\title{
Embryos Arising from Apronuclear (OPN) and Unipronuclear (1PN) Have Similar Euploidy Rates with Those from 2PN and Should be Considered for Transfer
}

\author{
Adelle Yun Xin Lim, Colin Soon Soo Lee \\ IVF Laboratory, Alpha Fertility Centre, Petaling Jaya, Selangor 47810, Malaysia
}

\begin{abstract}
Background: Fertilisation assessment is routinely made at $16-18$ hours post-ICSI and 18-20 hours post-insemination. However, the absence of pronuclei (PN) during standard fertilisation assessment does not necessarily indicate fertilisation failure. The aim of this study is to assess the chromosomal status of blastocysts derived from 0PN and 1PN zygotes as well as to assess the clinical outcome after transfer of such embryos.

Methods: In this study, we use microarray comparative genomic hybridisation (MaCGH) or next generation sequencing (NGS) to analyse the chromosomal status of 271 blastocysts (204 from 2PN, 41 from 0PN, 26 from 1PN) obtained from 42 patients who underwent conventional IVF (cIVF) and ICSI cycles with preimplantation genetic testing for aneuploidy (PGT-A).

Results: Euploidy was confirmed in 126 (126/204; 61.8\%), 31 (31/41; 75.6\%) and 18 (18/26; 69.2\%) 2PN-, 0PN- and 1PN-derived blastocysts respectively while the remaining 96 blastocysts displayed various chromosomal abnormalities. A Y-chromosome was observed in OPN-derived blastocysts $(19 / 41 ; 46.3 \%)$ and $1 \mathrm{PN}$-derived blastocysts $(13 / 26 ; 50 \%)$, indicating that sperm had penetrated the oocyte and not due to parthenogenetic activation. Four euploid 0PN-derived blastocysts were transferred to 4 patients and 3 healthy live births were achieved. Four euploid 1PN-derived blastocysts were transferred to 4 patients and 1 on-going pregnancy was achieved. Conclusion(s): $0 \mathrm{PN}$ - and $1 \mathrm{PN}$-derived zygotes can be chromosomally normal and result in healthy live births. Such zygotes should not be discarded but instead be subjected to extended culture with PGT-A to ascertain the chromosomal and ploidy status and be considered for transfer.
\end{abstract}

Keywords: Apronuclear; Unipronuclear; Ploidy; Preimplantation Genetic Testing; PGT.

\section{INTRODUCTION}

Fertilisation is routinely assessed 16-18 hours post-ICSI and 18-20 hours post-insemination (Montag and van der Ven, 2001). Normal fertilisation is determined by the presence of $2 \mathrm{PN}$ within the oocyte with defined membranes and 2 polar bodies in the perivitelline space (Campbell and Fishel, 2015).

Fertilisation is a process which involves the fusion of maternal and paternal gametes to initiate the formation of a new individual. The events that occur during fertilisation include: (a) sperm penetration; (b) sperm-zona pellucida interaction; (c) sperm-oocyte fusion; (d) oocyte activation; (e) sperm nucleus decondensation; (f) male and female PN formation; (g) pronuclear migration to the centre of the ooplasm; and (h) association of parental chromosomes on the spindle and first cleavage division (Plachot, 2000). The male $\mathrm{PN}$ is formed first and shortly followed by the female PN. The female PN then migrates towards the male PN until the 2 abut in the centre of the oocyte followed by PN breakdown permitting subsequent cleavage. Formation of the male and female PN is commonly visible simultaneously, however a small percentage may form asynchronously (Yasuyuki et al., 2014).

The appearance of $2 \mathrm{PN}$ is important in an IVF cycle since the $\mathrm{PN}$ number is taken to reflect the ploidy status of a zygote. Two PN suggest diploidy, while 3 or more PN indicate polyploidy (Manor et al., 1996). The absence of PN (OPN) indicates failed fertilisation and $1 \mathrm{PN}$ suggests abnormal fertilisation (Feenan and Herbert, 2006). The current routine practice is that only embryos that showed normal fertilisation $(2 \mathrm{PN})$ are chosen for transfer. However, occasionally there are no $2 \mathrm{PN}$ embryos available for transfer. Some oocytes without evidence of fertilisation such as $0 \mathrm{PN}$ and $1 \mathrm{PN}$ continue to cleave and form morphologically normal blastocysts and are indistinguishable from 2PN embryos. Such $0 \mathrm{PN}$ and $1 \mathrm{PN}$ embryos have been the subject of investigation but generally, IVF centres are uncertain whether such embryos with normal morphology should be used for transfer.

() 2019 by the Asia Pacific Initiative on Reproduction (ASPIRE) and World Scientific Publishing Co. Pte. Ltd. 2 Open Access article under the CC BY-NC-ND license (http://creativecommons.org/licenses/by-nc-nd/4.0/).

Received 30 January 2019; Accepted 30 May 2019; Published 13 June 2019

Correspondence should be addressed to: Dr. Adelle Yun Xin Lim, IVF Laboratory, Alpha Fertility Centre, 31, Level 2, Jalan PJU 5/6, Dataran Sunway, Kota Damansara, 47810 Petaling Jaya, Selangor, Malaysia. Email: adellelim@alphafertilitycentre.com 
The transfer of embryos deriving from $0 \mathrm{PN}$ and $1 \mathrm{PN}$ zygotes is rare but normal pregnancies as well as live births have been reported (Burney et al., 2008; Dasig et al., 2004; Gras and Trounson, 1999; Khoo et al., 2007; Lim et al., 2015; Lim et al., 2016; Manor et al., 1996; Noyes et al., 2008; Staessen et al., 1993). Analyses of chromosomal status in $0 \mathrm{PN}$ and $1 \mathrm{PN}$ embryos have been reported using fluorescence in situ hybridisation (FISH) on selected chromosome number mainly 13, 18, 21, X and Y (Khoo et al., 2007; Manor et al., 1996; Noyes, 2008; Staessen and van Steirteghem, 1997). We have previously reported complete screening of 23 sets of chromosomes in 0PN and 1PN embryos (Lee et al., 2013; Lee et al., 2015; Low et al., 2015).

Partly due to unavailability in most IVF centres for screening on all 23 chromosomes on $0 \mathrm{PN}$ and $1 \mathrm{PN}$ embryos, the chromosomal status of these embryos remains questionable and therefore are not considered for transfer. In this study, we screened all 23 sets of chromosomes in $0 \mathrm{PN}$ - and $1 \mathrm{PN}$-derived blastocysts in patients who underwent conventional IVF (cIVF) and ICSI cycles with PGT-A.

\section{MATERIALS AND METHODS}

\section{Patients and cycles}

A total of 42 patients undergoing IVF treatments (including cIVF, ICSI and combined conventional IVF and ICSI) with PGT-A who had either $0 \mathrm{PN}$ - and $1 \mathrm{PN}$-derived blastocysts were included in this study between November 2013 and November 2016. Cases with known altered karyotype of inversion and translocation; and indeterminate biopsies were excluded from this study. These 42 patients underwent controlled ovarian hyperstimulation with GnRH agonist or $\mathrm{GnRH}$ antagonist protocol. Ovulation was triggered by human chorionic gonadotrophin (hCG) when at least one follicle was $\geq 20 \mathrm{~mm}$ in diameter. Oocyte retrieval was performed 36.5-37.0 hours later under ultrasound-guided transvaginal aspiration.

\section{Laboratory}

For conventional IVF (cIVF), oocytes were inseminated 3-4 hours after oocyte retrieval. Patient's husband's spermatozoa were processed by a standard density gradient centrifugation and swim-up method with 250,000 motile spermatozoa loaded into each insemination well containing 5 cumulus-oocyte-complexes (COC). The insemination dish was incubated for at least 2 hours. After 2 hours, the oocytes were individually cultured in a day 0 dish; fertilisation assessment was done the following day between 18 and 20 hours post-insemination. For ICSI, cumulus cells from the oocytes were removed by denudation 2 hours after oocyte retrieval and ICSI (PIEZO-ICSI, Primetech, Japan) was performed as described elsewhere (Yap et al., 2015). Fertilisation was assessed the following day between 16 and 18 hours post-injection. In combined cIVF and ICSI cycles, half of the retrieved oocytes were subjected to CIVF and the other half were subjected to ICSI. After fertilisation assessment, $0 \mathrm{PN}$ - and $1 \mathrm{PN}$-derived zygotes were divided according to its respective $\mathrm{PN}$ status and were sequentially cultured to blastocyst stage.

\section{Biopsy and PGS}

Assisted hatching was done on all cleaved $2 \mathrm{PN}-, 0 \mathrm{PN}$ - and $1 \mathrm{PN}-$ derived zygotes on day 3. Blastocysts with at least a $3 \mathrm{BB}$ grade (Gardner's System) (Schoolcraft et al., 1999) were selected for trophectoderm (TE) cell biopsy on day 5 and/or 6. Three to 5 TE cells were dissected from each blastocyst using laser ablation with a non-contact Class I laser diode of $400 \mu$ s pulses (ZILOS-tk, Hamilton Thorne, USA) (Yang et al., 2012). Harvested TE cells were loaded into PCR tubes with $2.5 \mu \mathrm{l}$ of phosphate buffered saline (1× PBS) (Yang et al., 2011). All biopsied blastocysts were vitrified on either day 5 or day 6, depending on the day of biopsy using Cryotec Method (Cryotech, Japan).

The biopsied cells of each blastocyst were screened using microarray comparative genomic hybridisation (MaCGH) or next generation sequencing (NGS) to determine the chromosomal status of each blastocyst. For MaCGH, the biopsied cells were amplified, labelled, hybridised and analysed according to the manufacturer's protocol (BlueGnome, United Kingdom). For NGS, the biopsied cells were amplified, labelled and sequenced according to the manufacturer's protocol (Thermo Fisher, USA).

\section{Statistical analysis}

Comparison groups were established to assess whether the formation of morphologically normal blastocysts derived from $0 \mathrm{PN}$ and $1 \mathrm{PN}$ zygote exhibit similar euploidy and aneuploidy rates when compared with blastocysts derived from 2PN embryos in the cIVF and ICSI group. Statistical analysis was done using two-tailed Fisher's exact test. Statistical significance was defined as a $\mathrm{p}<0.05$.

\section{Frozen-thawed blastocyst transfer cycle (FBT)}

Hormone replacement treatment protocol was used in all FBT cycles. The proliferative phase was induced by taking oestrogen tablets (Progynova) for 13-15 days. After confirming the lining of the endometrium has developed sufficiently, daily administration of progesterone was initiated, and blastocyst transfer was performed on 5.5 day of progesterone administration. Warming of the blastocysts were performed using the Cryotec Method (Cryotech, Japan). Both oestrogen and progesterone were continued until the day of pregnancy test. Serum beta hCG was assessed 9-14 days after the day of embryo transfer. If tested positive, oestrogen and progesterone were continued for several weeks depending on physician's protocol. Pregnancy and number of gestational sacs were confirmed using transvaginal ultrasound.

\section{RESULTS}

A summary describing the IVF cycles that resulted in $0 \mathrm{PN}$ - and $1 \mathrm{PN}-$ derived blastocysts were outlined in Table 1 . The mean maternal age of the 42 patients was 31.7 years old. The number of oocytes retrieved and injected/inseminated was 943 and 797 respectively. A total of 271 blastocysts were biopsied for PGT, of which 204 blastocysts were derived from $2 \mathrm{PN}, 41$ derived from $0 \mathrm{PN}$ and 26 derived from $1 \mathrm{PN}$ respectively. One hundred and twenty-six $2 \mathrm{PN}$-derived blastocysts (61.8\%), $310 \mathrm{PN}$-derived blastocysts (75.6\%) and $181 \mathrm{PN}$-derived blastocysts $(69.2 \%)$ were tested to be euploid. The euploidy and aneuploidy rates were comparable between $2 \mathrm{PN}-, 0 \mathrm{PN}$ - and $1 \mathrm{PN}-$ derived blastocysts ( $\mathrm{p}>0.05)$.

Of the 0PN- and 1PN-derived blastocysts, 19 (19/41; 46.3\%) OPN-blastocysts and $131 \mathrm{PN}$-blastocysts (13/26; 50\%) displayed a Y-chromosome signal, indicating that sperm had penetrated the

Table 1. Summary of treatment cycles.

\begin{tabular}{lllll}
\hline & \multicolumn{1}{c}{ 2PN } & \multicolumn{1}{c}{ 0PN } & \multicolumn{1}{c}{ 1PN } & >2PN \\
\hline No. of zygotes (\%) & $474(59.5)$ & $201(25.2)$ & $51(6.4)$ & $28(3.5)$ \\
No. of blastocysts biopsied & 204 & 41 & 26 & - \\
No. of euploid (\%) & $126(61.8)$ & $31(75.6)$ & $18(69.2)$ & - \\
No. of aneuploid (\%) & $78(38.2)$ & $10(24.4)$ & $8(30.8)$ & - \\
$\begin{array}{l}\text { No. of single aneuploidy (\%) } \\
\begin{array}{l}\text { No. of multiple chromosomal } \\
\text { abnormalities (\%) }\end{array}\end{array}$ & $50(24.5)$ & $5(12.2)$ & $7(26.9)$ & - \\
\hline
\end{tabular}


Table 2. Summary of sex chromosome abnormalities.

\begin{tabular}{llll}
\hline Sex chromosome & \multicolumn{1}{c}{ 0PN } & \multicolumn{1}{c}{ 1PN } & Total \\
\hline Normal XX (\%) & $21(52.2)$ & $12(46.2)$ & $33(49.3)$ \\
Normal XY (\%) & $16(39.0)$ & $13(48.1)$ & $29(43.3)$ \\
XO (\%) & $1(2.4)$ & 0 & $1(1.5)$ \\
OY (\%) & 0 & $1(3.8)$ & $1(1.5)$ \\
XXY (\%) & $3(7.3)$ & 0 & $3(4.5)$ \\
Displayed Y (\%) & $19(46.3)$ & $13(50.0)$ & $32(47.8)$ \\
\hline
\end{tabular}

oocyte and not due to parthenogenetic activation (Table 2). Of these, 16 out of the $190 \mathrm{PN}$ - and all $131 \mathrm{PN}-\mathrm{Y}$ bearing blastocysts were euploid. One $0 \mathrm{PN}$-derived blastocyst $(\mathrm{XO})$ and one $1 \mathrm{PN}$-derived blastocyst (OY) were haploid; $7.3 \%(3 / 41)$ of the $0 \mathrm{PN}$-derived blastocysts were triploid (XXY). No triploid was seen in $1 \mathrm{PN}$ derived blastocyst group.

Tables 3 and 4 outlined the PGT-A results and outcome measures for all $0 \mathrm{PN}$ - and $1 \mathrm{PN}$-derived blastocysts. From the 49 $0 \mathrm{PN}$ - and $1 \mathrm{PN}$-derived blastocysts that were tested to be euploid, 11 of which were transferred to 11 patients: 4 patients had 0PN single blastocyst transfer (SBT), 4 patients had 1PN SBT, 2 patients had combined $2 \mathrm{PN}+0 \mathrm{PN}$ double blastocysts transfer (DBT) and 1 patient had combined $2 \mathrm{PN}+1 \mathrm{PN}$ DBT. No prenatal diagnosis was performed.

Three healthy live births and 1 on-going pregnancy $(24+5$ at the time of writing) resulted from $40 \mathrm{PN} \mathrm{SBT}$ and $41 \mathrm{PN}$ SBT respectively. All 4 pregnancies achieved were singleton.

Two live births resulted from $2 \mathrm{PN}+0 \mathrm{PN}$ DBT and both were singleton pregnancies. Therefore, we cannot confirm the PN origin of the foetus in these 2 live births.

\section{DISCUSSION}

Fertilisation assessment is routinely made at $16-18$ hours post-ICSI and $18-20$ hours post-cIVF, but $2 \mathrm{PN}$ can be detected as early as 5 hours post-insemination (Trounson and Osborn, 1993). Nagy et al. (1998) revealed that the appearance of 2PN was observed 6 hours post-ICSI and 8 hours post-IVF using ejaculated spermatozoa on human sibling oocytes. A preliminary study by Payne et al. (1997) described how normal fertilisation took place and that the timing of fertilisation events varied between oocytes. This time lapse study also showed male and female PNs were visible as early as 2 hours and 51 minutes post-ICSI and 63\% (20/32 normally fertilised oocytes) of the male and female PNs become visible simultaneously, with a further $25 \%$ (8/32 normally fertilised oocytes) becoming visible asynchronously within 30 minutes of each other. Another time lapse study (Yasuyuki et al., 2014) also demonstrated that the human male $\mathrm{PN}$ and female PN were formed as early as 6.6 hours and 6.8 hours respectively.

The absence of PN during standard fertilisation assessment does not necessarily indicate fertilisation failure (Elder and Dale, 2000). The observation that $20 \%$ of $0 \mathrm{PN}$ zygotes can progress to cleavage suggests either that observation of the PN can be missed due to syngamy before fertilisation check or that the PN only formed after the fertilisation check (Feenan and Herbert, 2006). Manor et al. showed 57\% of OPN embryos were normal diploid, 30\% polyploid and/or mosaic and $13 \%$ aneuploid following fluorescent in situ hybridisation (FISH) assay using X, Y, 13, 18 and 21 probes and the transfer of $0 \mathrm{PN}$ embryos had resulted in one on-going pregnancy and a normal female foetus $(46, \mathrm{XX})$ was documented
Table 3. PGS results and outcome measures of 0PN-derived blastocysts.

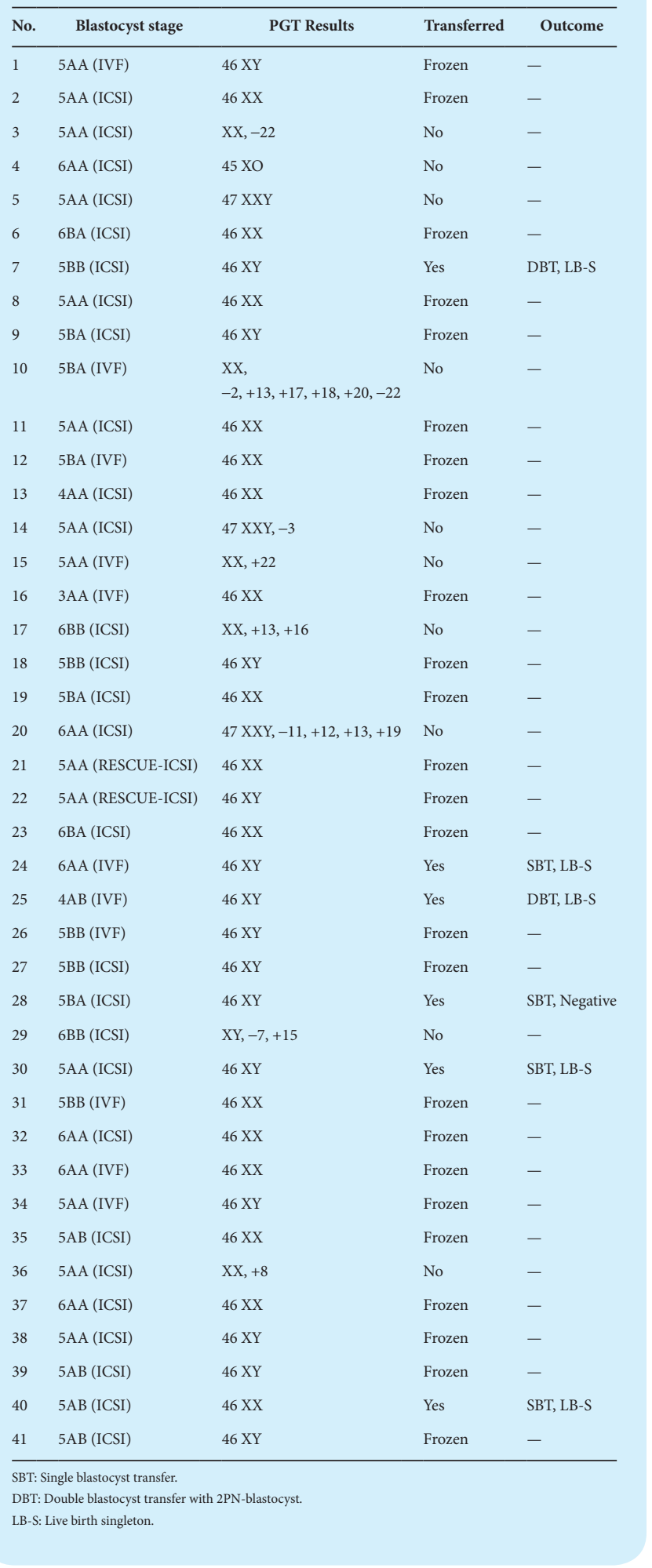

by amniocentesis (Manor et al., 1996). Consistent with this, Khoo et al. reported a successful on-going pregnancy after the transfer of a seven-cell embryo derived from a 0PN zygote, following FISH analysis $(13,18,21, \mathrm{X}$ and $\mathrm{Y})$. The chromosomal status was further confirmed by amniocentesis displaying a karyotype of 46XY (Khoo et al., 2007). Burney et al. (2008) also reported the birth of a healthy infant originating from a $0 \mathrm{PN}$ zygote.

Unipronuclear zygotes obtained following cIVF or ICSI may be due to oocyte parthenogenetic activation, possible fusion of male 
Table 4. Chromosomal status and outcome measures of $1 \mathrm{PN}$-derived blastocysts.

\begin{tabular}{|c|c|c|c|c|}
\hline No. & Blastocyst stage & PGT Results & Transferred & Outcome \\
\hline 1 & 5AA (IVF) & $46 \mathrm{XX}$ & Frozen & - \\
\hline 2 & $3 \mathrm{AB}(\mathrm{IVF})$ & $\mathrm{XY},+4$ & No & - \\
\hline 3 & 5AA (IVF) & $46 \mathrm{XX}$ & Frozen & - \\
\hline 4 & $5 \mathrm{AB}(\mathrm{IVF})$ & $\mathrm{XY},+5$ & No & - \\
\hline 5 & $4 \mathrm{AB}$ (IVF) & $46 \mathrm{XY}$ & Frozen & - \\
\hline 6 & 4AB (IVF) & $46 \mathrm{XY}$ & Frozen & - \\
\hline 7 & $5 \mathrm{AB}(\mathrm{IVF})$ & $\mathrm{XY},+3$ & No & - \\
\hline 8 & $5 \mathrm{AB}(\mathrm{IVF})$ & $\mathrm{XX},+11$ & No & - \\
\hline 9 & $5 \mathrm{AB}(\mathrm{IVF})$ & $\mathrm{XY},+21$ & No & - \\
\hline 10 & $6 \mathrm{AB}$ (IVF) & $46 \mathrm{XX}$ & Frozen & - \\
\hline 11 & 5AA (ICSI) & $\mathrm{XX},+16$ & No & - \\
\hline 12 & $5 \mathrm{AB}(\mathrm{IVF})$ & $46 \mathrm{XX}$ & Frozen & - \\
\hline 13 & $5 \mathrm{AB}(\mathrm{ICSI})$ & $46 \mathrm{XX}$ & Frozen & - \\
\hline 14 & $5 \mathrm{AB}(\mathrm{ICSI})$ & $46 \mathrm{XX}$ & Frozen & - \\
\hline 15 & 5AA (ICSI) & $46 \mathrm{XY}$ & Frozen & - \\
\hline 16 & $5 \mathrm{AB}(\mathrm{ICSI})$ & $46 \mathrm{XY}$ & Frozen & - \\
\hline 17 & $6 \mathrm{AB}(\mathrm{ICSI})$ & $46 \mathrm{XX}$ & Frozen & - \\
\hline 18 & $5 \mathrm{AB}(\mathrm{ICSI})$ & $46 \mathrm{XX}$ & Yes & SBT, Negative \\
\hline 19 & $5 \mathrm{AB}(\mathrm{ICSI})$ & $46 \mathrm{XX}$ & Yes & SBT, Negative \\
\hline 20 & 5BA (ICSI) & $46 \mathrm{XY}$ & Frozen & - \\
\hline 21 & 5BA (ICSI) & $45 \mathrm{OY}$ & No & - \\
\hline 22 & 6BB (ICSI) & $46 \mathrm{XX}$ & Frozen & - \\
\hline 23 & $5 \mathrm{AB}(\mathrm{ICSI})$ & $\begin{array}{l}\mathrm{XY},+7,+9,+14 \\
+16,+19,+20 \\
-22\end{array}$ & No & - \\
\hline 24 & 6AA (ICSI) & $46 \mathrm{XY}$ & Yes & DBT, Negative \\
\hline 25 & $5 \mathrm{AB}$ (ICSI) & $46 \mathrm{XY}$ & Yes & SBT, on-going \\
\hline 26 & $5 \mathrm{AB}(\mathrm{ICSI})$ & $46 \mathrm{XY}$ & Yes & SBT, Negative \\
\hline
\end{tabular}

and female PN or asynchronous PN formation (Gras and Trounson, 1999). The development of parthenogenetic oocytes is impaired after activation, thus leading to early embryo arrest usually at the 8-cell stage (Winston et al., 1991) and failure to activate the embryonic genome for further embryonic development. Chromosomal studies revealed that $45-48 \%$ of diploid $1 \mathrm{PN}$ zygotes are in fact fertilised displaying Y signals and were not due to parthenogenetic activation. In a study by Plachot and Crozet (1992), it was observed that $77 \%$ of $1 \mathrm{PN}$ oocytes develop past early cell divisions, of these, $14.3 \%$ are able to form morphologically normal blastocysts and are indistinguishable from $2 \mathrm{PN}$ embryos suggesting that these $1 \mathrm{PN}$ oocytes may in fact be derived from fertilised oocytes with a diploid complement. It was suggested that such embryos may be the product of $2 \mathrm{PN}$ sharing the same PN envelope after the fusion of male and female PN (Tessarik and Mendoza, 1996). Asynchronous formation of $\mathrm{PN}$ was also observed in $25 \%$ of $1 \mathrm{PN}$ zygotes, where such zygotes develop the second PN when reassessed 4 hours after the initial fertilisation assessment (Staessen et al., 1993). Subsequently, Payne et al. (1997) affirmed this theory finding 38\% (12/32) of fertilised oocytes developed PN asynchronously with the male PN appearing before the female $\mathrm{PN}$, with the average time between the appearance of the two PN being 31 minutes. By extending in-vitro culture to day 5 or 6 , morphologically normal blastocysts may possibly be used as an indicator of fertilisation and may be selected for transfer when insufficient 2PN embryos are available (Gras and Trounson, 1999).
A study by Noyes (2008) demonstrated that at 18 hours postinsemination, the zygote distribution was $19 \% 0 \mathrm{PN}, 4 \% 1 \mathrm{PN}$ and $69 \% 2 \mathrm{PN}$ and $8 \% 3 \mathrm{PN}$ out of 1908 embryos assessed. From these, the euploid karyotypes for $0 \mathrm{PN}, 1 \mathrm{PN}$ and $2 \mathrm{PN}$ were $3 \%, 5 \%$ and $19 \%$ respectively following FISH analysis (X, Y, 13, 15, 16, 17, 18, 21 and 22$)(\mathrm{p}=0.015$ for $1 \mathrm{PN}$ vs. $2 \mathrm{PN}$; no statistical significance for 0PN vs. 2PN). In 2013, Lee et al. (2013) at Alpha Fertility Centre showed that OPN and 1PN embryos following ICSI that develop to the cleavage stage have a euploidy rate similar to embryos resulting from 2PN after microarray comparative genomic hybridisation (MaCGH). Again, Lee et al. (2015) reported 273 blastocysts following cIVF were analysed using MaCGH and from these, $68.8 \%$ of $0 \mathrm{PN}$ blastocysts, $52.9 \%$ of $1 \mathrm{PN}$ blastocysts and $51.7 \% 2 \mathrm{PN}$ blastocysts were euploid. In the same year, Low et al. (2015) from Alpha Fertility Centre also demonstrated comparable euploidy rates ( $\mathrm{p}>0.05$ ) among $0 \mathrm{PN}, 1 \mathrm{PN}$ and $2 \mathrm{PN}$ day 3 embryos following cIVF using MaCGH.

Since 49 OPN- and 1PN-derived blastocysts (49 out of 67) in our study were found to be euploid, our results document the possibility that accelerated disappearance of the PN membrane or asynchronous PN formation following either cIVF or ICSI, do not necessarily reflect aneuploidy or failed fertilisation. This study also suggests that if $0 \mathrm{PN}$ and $1 \mathrm{PN}$ zygotes that can form morphologically normal blastocysts do not necessarily reflect abnormal embryo development and can be euploid. On the other hand, the pregnancies achieved by transferring $0 \mathrm{PN}$ - and $1 \mathrm{PN}$-derived blastocysts establishes the ability of such blastocysts to implant.

OPN and 1PN transfers are controversial and many IVF centres prefer not to transfer such embryos and discard them based on the universal fertilisation assessment timings. Thus, some patients, especially patients with decreased ovarian reserve or older patients may missed the chance for pregnancy when there is a lack of normal 2PN embryos for transfer.

Although blastocyst culture facilitates the selection of morphologically normal $\mathrm{OPN}$ and $1 \mathrm{PN}$ for transfer, clinics may want to impose policies requiring PGT-A to screen $0 \mathrm{PN}$ and $1 \mathrm{PN}$ derived embryos to determine their chromosome constitution prior to transfer. In addition to that, time-lapse monitoring can be used to allow a detailed observation of second polar body extrusion and pronuclear formation. Transferring of $0 \mathrm{PN}$ and $1 \mathrm{PN}$ embryos should only be considered for transfer especially where there is a lack of 2PN-derived embryos. On-going pregnancies resulting from $0 \mathrm{PN}$ or 1PN euploid blastocyst transfers should be followed by non-invasive prenatal testing (NIPT) at 9-10 weeks and by prenatal diagnosis such as chorionic villus sampling (CVS) at 11-12 weeks or amniocentesis at $15-16$ weeks to confirm a chromosomally normal foetus. Also, patients should be counselled appropriately, and informed decisions made about the safety of transferring these embryos.

\section{ACKNOWLEDGEMENTS}

We would like to thank Dr John Keith for his critical reading of the manuscript. The authors also thank all the IVF staff, especially the embryologists and geneticists. This research did not receive any specific grant from funding agencies in the public, commercial, or not-for-profit sectors.

\section{REFERENCES}

Burney RO, Gebhardt J, Shu Y, Behr B, Westphal LM. Normal pregnancy resulting from a non-pronuclear oocyte at the time of examination for fertilization. Clin Exp Obstet Gynecol. 2008;35:170-1. 
Campbell A, Fishel S. Atlas of Time Lapse Embryology. Boca Raton: CRC Press; 2015: p. 25.

Dasig D, Lyon J, Behr B, Milki AA. Monozygotic twin birth after the transfer of a cleavage stage embryo resulting from a single pronucleated oocyte. J Assist Reprod Genet. 2004;21:427-9.

Elder K, Dale B. In Vitro Fertilization, 2nd ed. Cambridge University Press; 2000: pp. 59-63.

Feenan K, Herbert M. Can 'abnormally' fertilized zygotes give rise to viable embryos? Hum Fertil. 2006;9(3):157-69.

Gras L, Trounson AO. Pregnancy and birth resulting from transfer of a blastocyst observed to have one pronucleus at the time of examination for fertilization. Hum Reprod. 1999;14(7):1869-70.

Khoo G, Lee CSS, Wong PS, Lee SA, Keith J. To report a case with on-going pregnancy after the transfer of a seven cell embryo derived from an apronucleate zygote, following PGD-FISH. Poster presentation at the 7 th International Conference on Preimplantation Genetic Diagnosis International Society, Melbourne, Australia, 13-15 June 2007.

Lee CSS, Yap WY, Lim MW, Lim YX, Low SY, Lui WX. Euploidy rates of apronuclear (0PN) and unipronuclear (1PN) blastocysts fertilised using in vitro fertilization (IVF). Reprod BioMed Online. 2015;31(2):292.

Lee CSS, Yap WY, Low SY, Lim YX. Euploidy rates for day 3 apronuclear (OPN) and unipronuclear (1PN) embryos. Reprod BioMed Online. 2013;26:S36.

Lim YX, Lee CSS, Yap WY, Low SY, Lui WX, Lim MW. Live birth following frozen embryo transfer of a 1PN embryo after PGDMaCGH analysis. Reprod BioMed Online. 2015;31(2):293.

Lim YX, Yap WY, Keith J, Lee CSS. Case report: live twin birth following transfer of a $2 \mathrm{PN}$ and a $0 \mathrm{PN}$ embryo after PGD-MaCGH. Reprod BioMed Online. 2016;33(2):76

Low SY, Lee CSS, Lim MW, Yap WY, Lui WX, Lim YX. Euploidy rates of day 3 apronuclear (0PN) and unipronuclear (1PN) cleaved embryo arising from IVF. Reprod BioMed Online. 2015;31(2):294.

Manor D, Kol S, Lewit N, Lightman A, Stein D, Pillar M, ItskovitzEldor J. Undocumented embryos: do not trash them, FISH them. Hum Reprod. 1996;11(11):2502-6.

Montag M, van der Ven H. Evaluation of pronuclear morphology as the only selection criterion for further embryo culture and transfer: results of a prospective multicentre study. Hum Reprod. 2001;16:2384-9.

Nagy ZP, Janssenswillen C, Janssens R, DeVas A, Staessen C, van de Velde H, van Steirteghem AC. Timing of oocyte activation, pronucleus formation and cleavage in human after introcytoplasmic sperm injection (ICSI) with testicular spermatozoa and after ICSI or in-vitro fertilization on sibling oocytes with ejaculated spermatozoa. Hum Reprod. 1998;13:1606-12.
Noyes N. Embryo biopsy: the fate of abnormal pronuclear embryos. Reprod BioMed Online. 2008;17(6):782-8.

Payne D, Flaherty SP, Barry MF, Matthews CD. Preliminary observation on polar body extrusion and pronuclear formation in human oocytes using time-lapse video cinematography. Hum Reprod. 1997;12:532-41.

Plachot M. Fertilization. Hum Reprod. 2000;15(S4):19-30.

Plachot M, Crozet N. Fertilization abnormalities in human in-vitro fertilization. Hum Reprod. 1992;7:89-94.

Schoolcraft WB, Gardner DK, Lane M, Schlenker T, Hamilton F, Meldrum DR. Blastocyst culture and transfer: analysis of results and parameters affecting outcome in two in vitro fertilization programs. Fertil Steril. 1999;72:604-9.

Staessen C, Janssenswillen C, Devroey P, van Steirteghem A. Cytogenetic and morphological observations of single pronucleated human oocytes after in-vitro fertilization. Hum Reprod. 1993;8: 221-3.

Staessen C, van Steirteghem AC. The chromosomal constitution of embryos developing from abnormally fertilized oocytes after intracytoplasmic sperm injection and conventional in-vitro fertilization. Hum Reprod. 1997;12:321-7.

Tessarik J, Mendoza C. Spermatid injection into human oocytes. I. Laboratory techniques and special features of zygote development. Hum Reprod. 1996;11:772-9.

Trounson JC, Osborn J. In vitro fertilization and embryo development. In: Trounson AO, Gardner DK (editors). Handbook of In Vitro Fertilization. Boca Raton: CRC Press; 1993: p. 57-84.

Winston N, Johnson M, Pickering S, Braude P. Parthenogenetic activation and development of fresh and aged human oocytes. Fertil Steril. 1991;56:904-12.

Yang Z, Liu J, Collins GS, Salem S, Liu X, Lyle S, Peck AC, Sills ES, Salem RD. Selection of single blastocysts for fresh transfer via standard morphology assessment alone and with array CGH for good prognosis IVF patients: results from a randomized pilot study. Fertil Steril. 2012;5:24.

Yang Z, Salem S, Salem-Lyle S, Bayrak A, Salem RD. Trophectoderm cells derived from blastocyst biopsy are suitable for array CGH analysis of 24 chromosomes. Fertil Steril. 2011;95(S4):S23.

Yap WY, Lee CSS, Low SY, Lim YX, Lui WX, Lim MW. Chromosomal segregation during embryonic cellular division is not affected by PIEZO Intracytoplasmic Sperm Injection (PIEZO_ICSI). Reprod BioMed Online. 2015;31(2):303.

Yasuyuki M, Kyoko I, Keitaro Y, Kazuo M. Human embryonic behaviour observed with time-lapse cinematography. J Health Med Inform. 2014;5(1):143. 